\title{
Intrinsic MRI visualizes RF lesions within minutes after MR-guided ablation
}

\author{
Philippa Krahn ${ }^{2,1^{*}}$, Venkat Ramanan ${ }^{1}$, Labonny Biswas ${ }^{1}$, Robert Xu ${ }^{2,1}$, Jennifer Barry ${ }^{1}$, Nicolas Yak', \\ Kevan Anderson ${ }^{1,2}$, Sheldon Singh ${ }^{3}$, Mihaela Pop ${ }^{2,1}$, Graham A Wright ${ }^{1,2}$ \\ From 19th Annual SCMR Scientific Sessions \\ Los Angeles, CA, USA. 27-30 January 2016
}

\section{Background}

MR visualization of RF lesions is an application of growing interest with the potential for translation to clinical ablation procedures. In particular, intrinsic-contrast MRI avoids the dynamic contrast produced in typical Gd-based MRI, and may differentiate the reversible and irreversible thermal injury thought to be caused by RF ablation. This distinction is important for assessing the permanence of ablation to eradicate the substrate of ventricular tachycardia in structural heart disease. In this study we investigate the potential of intrinsic-contrast MRI to visualize the features of thermal injury and evolution of RF lesions that may occur immediately after ablation.

\section{Methods}

$8 \mathrm{RF}$ ablation lesions were created in vivo in 4 healthy pigs. Active real-time MR tracking [1] guided navigation of an MR-enabled catheter (Imricor Medical Systems) within the LV. During ablation, 30-40W was applied to the LV endocardium for 45-60s with catheter tip irrigation throughout. MR images were acquired repeatedly during the ensuing 1-2h, a time frame relevant to the length of clinical ablation procedures. The imaging protocol consisted primarily of $\mathrm{T}_{2}$-prepared b-SSFP for $\mathrm{T}_{2}$ mapping and IR-prepared $b$-SSFP. In $T_{2}$ maps, long- $T_{2}$ regions representative of inflamed, edematous tissue were delineated semi-automatically using a threshold of $\mathrm{T}_{2}=55 \mathrm{~ms}$, approximately $3 \mathrm{SD}$ above remote as per established methods describing edema in $\mathrm{T}_{2}$-weighted images [2]. Further, we manually delineated the core of RF lesions based on myocardial hyperenhancement in IR-SSFP images as in [3]. In all analysis, one-tailed t-test was used and $\mathrm{p}<0.05$ considered significant.

\section{Results}

In a subset of 4 lesions, the earliest $\mathrm{T}_{2}$-prep acquisition was 9 min post-ablation and clearly demonstrated edema at the ablation site. Overall, $\mathrm{T}_{2}$ in the edematous and remote tissue was $64.8 \mathrm{~ms}$ and $40.7 \mathrm{~ms}$ respectively. The edematous areas increased markedly with time postablation, by $89 \%$ on average. This trend was significant for the exemplary lesion in Figure 1, where $\mathrm{p}<0.05$ (rejecting the null hypothesis that the slope is 0 ), and edema size seemed to stabilize after $36 \mathrm{~min}$. In a second subset of 4 lesions, the earliest IR-SSFP acquisition was 3 min postablation and these images clearly demonstrated the lesion core. The exemplary lesion core in Figure 2 remained stable over time ( $>0.05$ indicating no change in the core area). Although limited by noise in initial images acquired with a surface coil, preliminary results support the stabilization of lesion core.

\section{Conclusions}

We successfully demonstrated the visualization of RF lesions using intrinsic-contrast MRI during a time frame spanning minutes to hours after ablation. The presence of edema is of particular interest as it is thought to temporarily alter myocardial excitability, confounding clinical tests used to confirm RF ablation procedural success. This valuable description of RF lesions could be integral in future ablation procedures performed concurrently with MRI feedback.

\footnotetext{
Authors' details

${ }^{1}$ Physical Sciences Platform, Sunnybrook Research Institute, Toronto, ON, Canada. ${ }^{2}$ Medical Biophysics, University of Toronto, Toronto, ON, Canada. ${ }^{3}$ Cardiology, Sunnybrook Health Sciences Centre, Toronto, ON, Canada.
} 

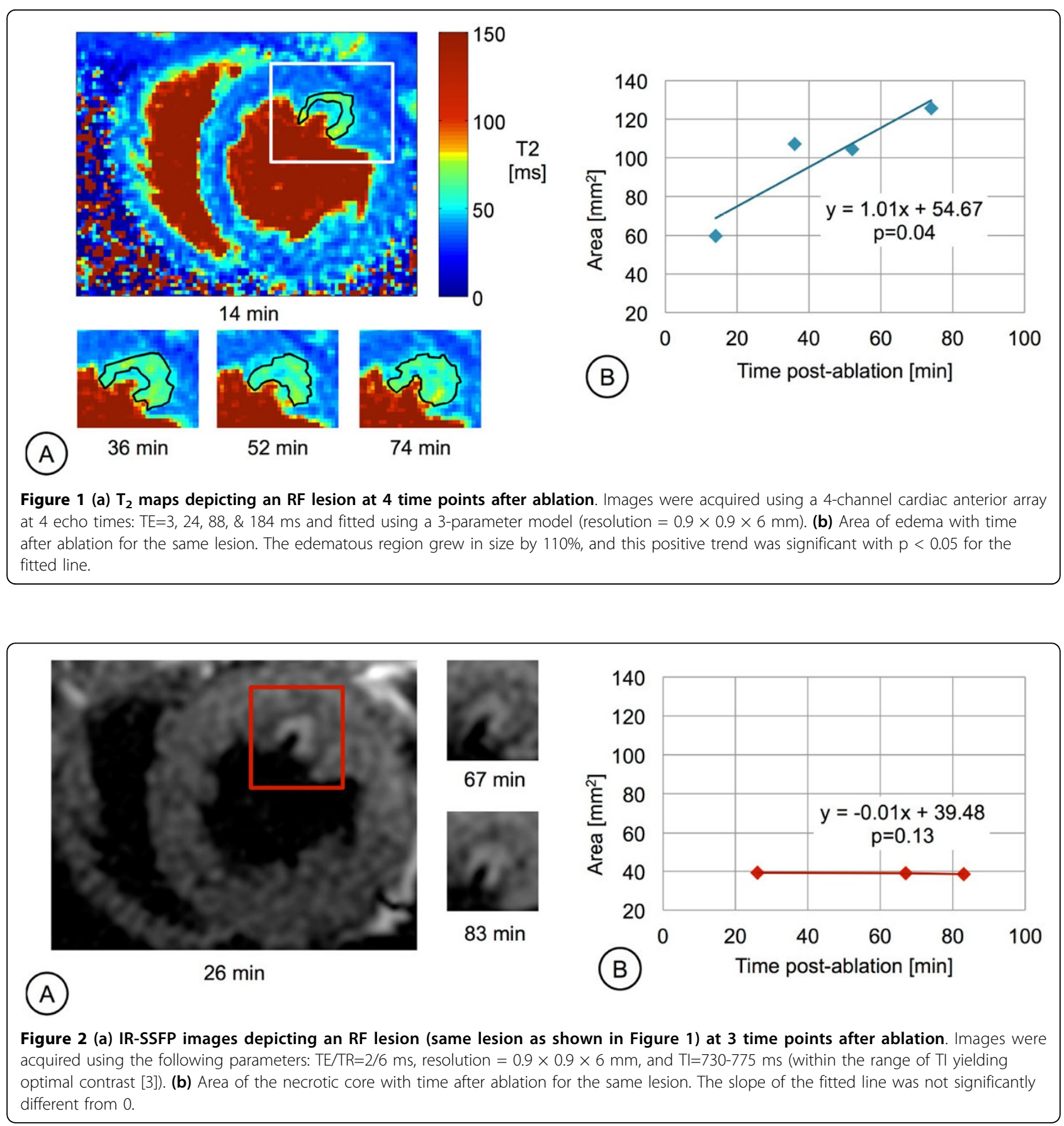

Published: 27 January 2016

doi:10.1186/1532-429X-18-S1-P206

Cite this article as: Krahn et al:: Intrinsic MRI visualizes RF lesions within minutes after MR-guided ablation. Journal of Cardiovascular Magnetic

Resonance 2016 18(Suppl 1):P206. 